\title{
Interpretation of non-MET+X ATLAS and CMS searches for dark matter scenarios
}

\author{
Claudia-Elisabeth Wulz; , on behalf of the ATLAS and CMS collaborations \\ Institute of High Energy Physics of the Austrian Academy of Sciences, Nikolsdorfergasse 18, \\ A-1050 Vienna, Austria \\ E-mail: claudia.wulz@eern.ch
}

\begin{abstract}
Dark matter searches by the ATLAS and CMS experiments at the CERN LHC in final states with no missing transverse energy are presented, using channels with dileptons and dijets, in particular. Through a study of dijet angular distributions the low-mass mediator regions have been explored for the first time. Most of the analyses were performed with data recorded at a centre-of-mass energy of $13 \mathrm{TeV}$.
\end{abstract}

An Alpine LHC Physics Summit (ALPS2018)

15-20 April, 2018

Obergurgl, Austria

${ }^{*}$ Speaker. 


\section{Introduction}

At colliders dark matter (DM) searches conventionally target weakly interacting massive particles (WIMPs) in channels with missing transverse energy (MET), labeled as "mono- $X$ " searches,

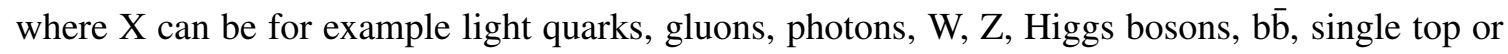
$\mathrm{t} \overline{\mathrm{t}}$. Searches for displaced DM, originating from the decay of long-lived particles, have also gained importance recently. In this presentation we however concentrate on searches with dileptons and dijets, with no significant MET in the final state, which extend the discovery range with respect to the classical searches. Results from proton-proton data recorded with the ATLAS [1] and CMS [2] experiments are presented.

Production of DM particles at the LHC can occur through decays. The lightest supersymmetric particle could thus appear in SUSY cascades. They may also be produced directly, for example through a Higgs or a Z' portal. Properties to be measured include the DM particle $\left(m_{\mathrm{DM}}\right)$ and mediator masses $\left(m_{\mathrm{Med}}\right)$, the couplings of the mediator to the DM particle $\left(g_{\mathrm{DM}}\right)$, to leptons $\left(g_{\ell}\right)$ and quarks $\left(g_{\mathrm{q}}\right)$, and the types of interactions (scalar, pseudoscalar, vector, axial-vector, tensor).

\section{Dileptons}

Dileptons in a model with a spin-1 high-mass vector or axial-vector mediator have been studied by CMS [3]. Five parameters define the production and decay rates of the mediator and the event kinematics: $m_{\mathrm{DM}}, m_{\mathrm{Med}}, g_{\mathrm{DM}}, g_{\ell}, g_{\mathrm{q}}$. Benchmark couplings illustrating the complementary strengths of the dilepton/dijet analyses have been chosen. With $g_{\text {DM }}$ set to $1, g_{\mathrm{q}}=0.1$ and $g_{\ell}=0.01$ are assumed for a vector mediator, and $g_{\mathrm{q}}=g_{\ell}=0.1$ for an axial-vector mediator. The electron selection requires two isolated electromagnetic clusters with $p_{\mathrm{T}}>35 \mathrm{GeV}$ and $|\eta|<1.44$ or 1.57 $<|\eta|<2.50$. At least one electron must be in the barrel region to reduce the QCD background, but dielectrons need not be oppositely charged. The muon selection requires at least two isolated

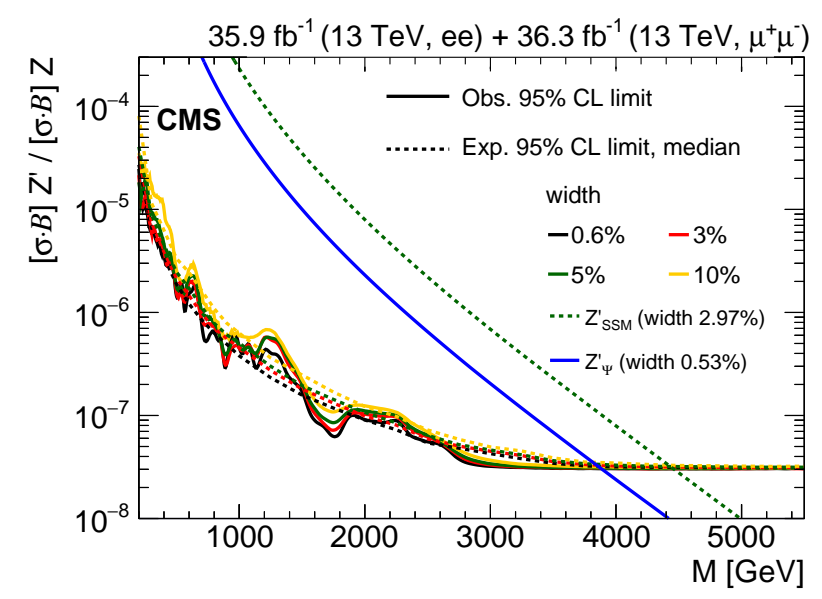

Figure 1: The upper limits at 95\% CL on the product of production cross section and branching fraction for a spin-1 resonance, for widths equal to $0.6,3,5$, and $10 \%$ of the resonance mass, relative to the product of production cross section and branching fraction for a $\mathrm{Z}$ boson, for the combination of dielectron and dimuon channels [3]. Theoretical predictions for the spin-1 Z' ${ }_{\text {SSM }}$ and Z' ${ }_{\psi}$ resonances are also shown. 
muon tracks with $p_{\mathrm{T}}>50 \mathrm{GeV}$ and $|\eta|<2$.4. Backgrounds are estimated from simulation (except misidentified leptons from QCD jets) and include the dominant Drell-Yan background, photons radiated from incoming protons $\left(\gamma \gamma \rightarrow \ell^{+} \ell^{-}\right.$, included in the Drell-Yan NNLO K-factor), and leptons from $\mathrm{t} \overline{\mathrm{t}}, \mathrm{tW}, \mathrm{WW}, \mathrm{WZ}, \mathrm{ZZ}$, and $\tau^{+} \tau^{-}$. Fig. 1 shows the $\mathrm{Z}^{\prime}$ production cross section times branching fraction relative to the $\mathrm{Z}$ boson. $\mathrm{Z}$ ' mass limits of $\mathrm{M}\left(\mathrm{Z}^{\prime}{ }_{\mathrm{SSM}}\right)>4.5 \mathrm{TeV}$ and $\mathrm{M}\left(\mathrm{Z}^{\prime}{ }_{\psi}\right)>$ $3.9 \mathrm{TeV}$ for the generalised sequential standard model [4] and the $\psi$ model based on the $E_{6}$ gauge group in grand unified theories [5] can be derived. Similar values have been obtained by ATLAS [6]. If $m_{\mathrm{DM}}$ is larger than $m_{\mathrm{Med}} / 2$ the mediator cannot decay to DM, and the leptonic branching fraction becomes sizable. For the vector mediator case the small lepton branching fraction limits the sensitivity to $m_{\mathrm{DM}}>m_{\mathrm{Med}} / 2$, as can be seen in Fig. 2 (left). This is an interesting range to probe, almost inaccessible to MET searches. For the axial-vector model, the leptonic couplings are large, therefore an exclusion is also possible for $m_{\mathrm{DM}}<m_{\mathrm{Med}} / 2$, as can be seen in Fig. 2 (right).
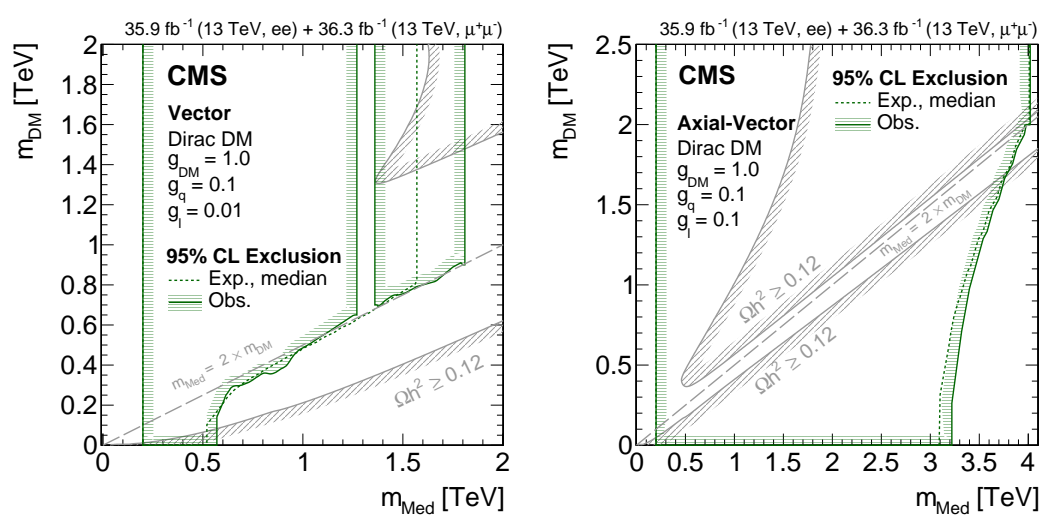

Figure 2: Limits at 95\% confidence level for the masses of the DM particle, which is assumed to be Dirac fermion, and its associated mediator [3], in a simplified model of DM production via a vector mediator (left), and via an axial-vector mediator (right).

\section{Dijets}

Dijet analyses have been used since the start of the LHC experiments to look for new highmass resonances. In dark matter searches low-mass mediator regions have now become accessible through the study of angular distributions and the use of special data acquisition methods, which allow recording large amounts of data, albeit with reduced stored event information. These methods go by the name of trigger-level jets in ATLAS, and data scouting in CMS. A low-mass dijet analysis has been performed by ATLAS in the mass range 450 to $1800 \mathrm{GeV}$, using trigger-level jets [7]. A new sliding-window fit to estimate the background has also been used, where the dijet spectra are fitted in smaller windows instead of the entire mass range. A bump hunter qualifies the statistical significance of any localised excess. Figure 3 (left) shows the limits on the coupling $g_{\mathrm{q}}$ as a function of the Z' mediator mass, demonstrating the extension of the mass reach to lower masses compared to the traditional dijet analyses. ATLAS has also searched for a boosted dijet signal in combination with a photon or a jet from initial state radiation [8]. The collimated decay products of the DM mediator are merged into a large-radius jet. Whilst one can trigger on the ISR jet or photon, 

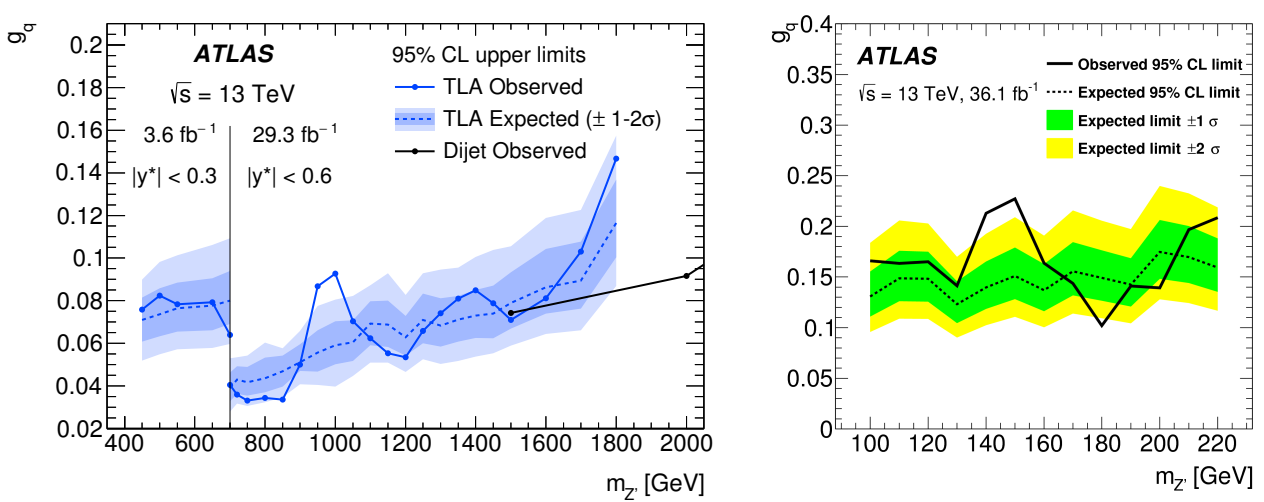

Figure 3: (Left) Observed and expected limits at 95\% confidence level on $g_{\mathrm{q}}$ as a function of the $\mathrm{Z}^{\prime}$ mass for the trigger-level jet analysis [7]. Couplings above the solid lines are excluded. (Right) Observed and expected limits for the boosted analysis at $95 \%$ confidence level on the coupling $g_{\mathrm{q}}$, for the combination of the ISR jet and photon channels [8].

substructure techniques have to be used to identify the quark pair. As Fig. 3 (right) shows, the mass reach for the mediator extends down to $100 \mathrm{GeV}$. A CMS analysis [9], where results from both boosted and resolved dijets are presented, found that mediator masses down to $50 \mathrm{GeV}$ can even be excluded for the chosen model, as can be seen from Figure 4 (left). A compilation of ATLAS, CMS, CDF, and UA2 results for a Z ' ${ }_{\mathrm{B}}[10]$ decaying to dijets at various centre-of-mass energies is presented in Fig. 4 (right) [11].
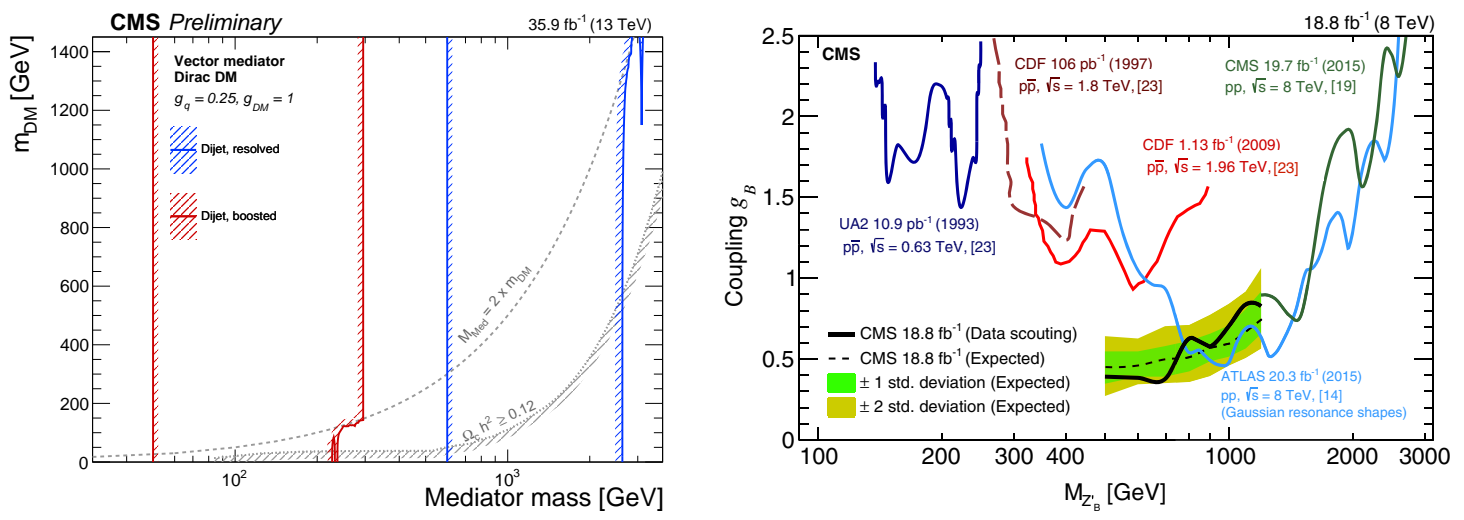

Figure 4: (Left) The 95\% CL observed (solid red) and expected (dashed red) excluded regions in the plane of dark matter particle mass versus mediator mass, for vector mediators [9]. A branching fraction of $100 \%$ is assumed for a leptophobic vector mediator decaying to dijets. The exclusion is computed for a quark coupling choice $g_{\mathrm{q}}=0.25$ and for a dark matter coupling $g_{\mathrm{DM}}=1$. The excluded regions from the dijet resolved analysis (blue lines) using early 2016 data are also shown. Results are compared to constraints from the cosmological relic density of DM (light gray) determined from astrophysical measurements. (Right) Observed CMS 95\% CL upper limits on the coupling $g_{\mathrm{B}}$ of a hypothetical leptophobic resonance $Z_{\text {' }}$, decaying to dijets as a function of its mass. The results are compared to results from similar searches at different experiments and collider energies [11].

In contrast to standard dijet searches, dijet angular distributions are sensitive to wide reso- 
nances, and also to nonresonant production. They reflect the dynamics of the scattering process, without strong dependence on parton distribution functions. Rutherford scattering leads to a flat distribution of the variable $\chi_{\text {dijet }}$, which is defined as

$$
\chi_{\text {dijet }}=\frac{1+\cos \theta^{*}}{1-\cos \theta^{*}}=\exp \left(\left|y_{1}-y_{2}\right|\right),
$$

where $y_{1}$ and $y_{2}$ are the rapidities of the two jets with the highest transverse momenta, and $\theta^{*}$ is the polar scattering angle in the parton-parton centre-of-mass frame. An excess of events at small $\chi_{\text {dijet }}$ could indicate a contribution from beyond-standard-model physics. A CMS analysis [12] studied normalized $\chi_{\text {dijet }}$ distributions, unfolded to particle level and compared to NLO predictions with electroweak corrections. Results along with predictions for three different DM masses are shown in Fig. 5. No excess has been found.
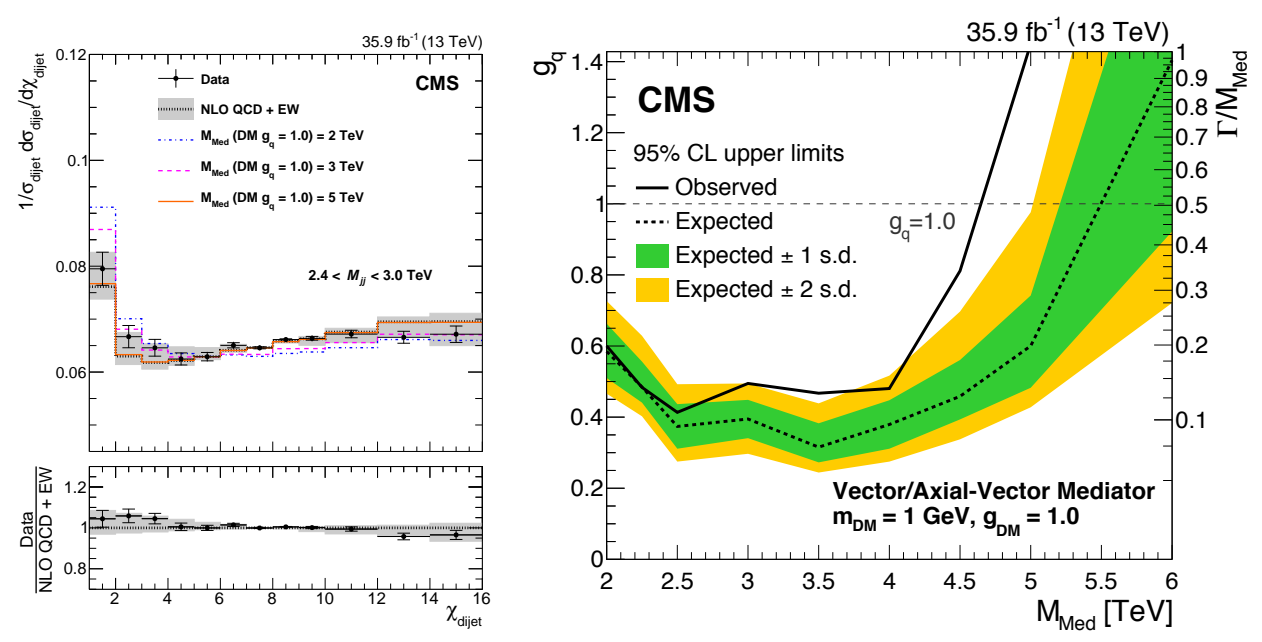

Figure 5: (Left) Normalized $\chi_{\text {dijet }}$ distribution in dijet mass bin $2.4<\mathrm{M}_{\mathrm{jj}}<3.0 \mathrm{TeV}$ [12]. Unfolded data are compared to NLO predictions (black dotted line). The lower panel shows the ratio of the unfolded data distributions and NLO predictions. (Right) The 95\% CL upper limits on the quark coupling $g_{\mathrm{q}}$, as a function of mass, for an axial-vector or vector DM mediator with $g_{\mathrm{DM}}=1.0$ and $m_{\mathrm{DM}}=1 \mathrm{GeV}$ [12]. The observed limits (solid), expected limits (dashed) and the variation in the expected limit at the 1 and 2 standard deviation levels (shaded bands) are shown. A dotted horizontal line shows the coupling strength for a benchmark DM mediator with $g_{\mathrm{q}}=1.0$. The corresponding limits on the width of the mediators are shown on the vertical axis on the right-hand side of the figure.

\section{Summary}

Dark matter summary plots for ATLAS and CMS are shown in Figs. 6 and 7. The ATLAS exclusion plot (Fig. 6) shows an updated version [13] of the one presented at the conference. It can be seen in particular that the gap in the low-mass mediator region between 300 and $500 \mathrm{GeV}$ has been filled by the boosted-regime analysis of dijets with accompanying initial state radiation. ATLAS and CMS limits on the universal coupling between a leptophobic Z' boson and quarks are shown in Fig. 7, both plots of which have also been updated since the conference. It can be seen that a large mediator mass range has been covered by the different analyses of the experiments. In 


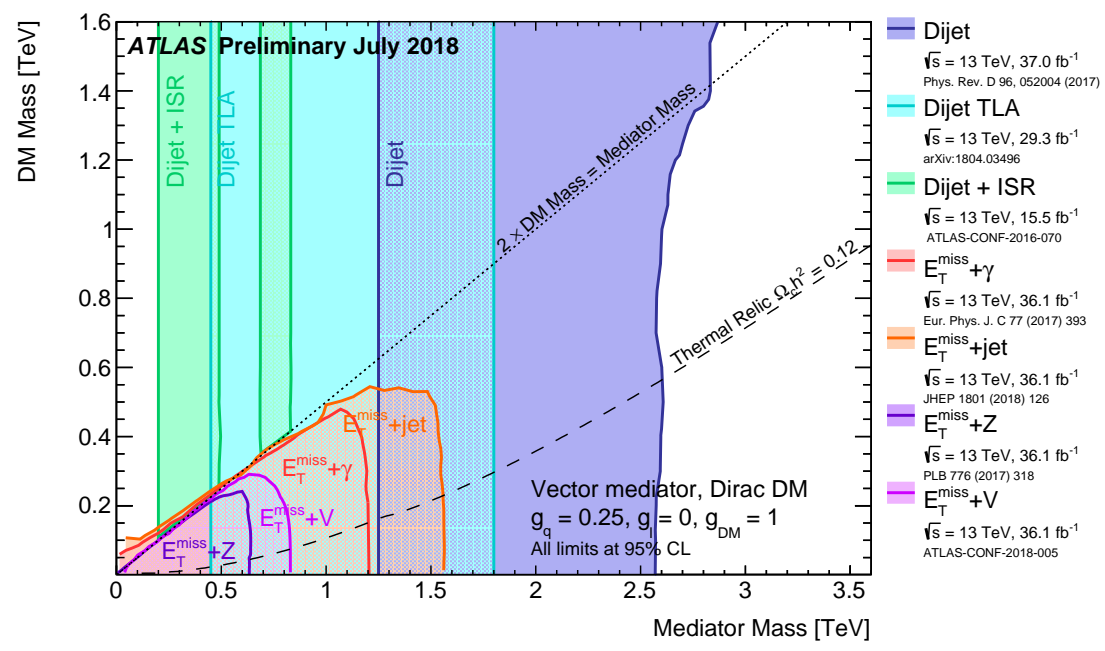

Figure 6: Regions in a dark matter mass-mediator mass plane excluded at 95\% CL by a selection of ATLAS dark matter searches [13], for one possible interaction between the Standard Model and dark matter, the leptophobic vector mediator as described in Ref. [14]. The results rely on $13 \mathrm{TeV}$ data collected in 2015 and 2016. A dotted curve indicates the kinematic threshold where the mediator can decay on-shell into dark matter.
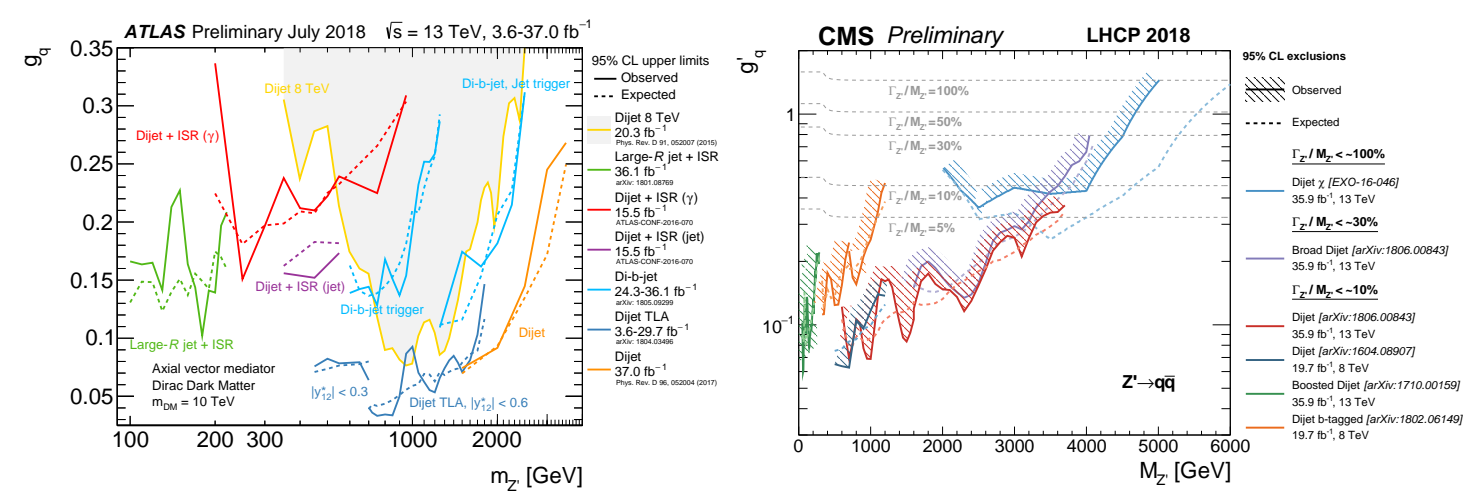

Figure 7: (Left) Summary plot of ATLAS bounds in the coupling-mediator mass plane from dijet searches using 2012, 2015 and 2016 data [15]. The 95\% CL upper limits are obtained from seven ATLAS analyses on the coupling $g_{\mathrm{q}}$ as a function of the $\mathrm{Z}$ ' resonance mass for the leptophobic Z' model of an axial-vector mediator between quarks and Dirac dark matter [16]. The expected limits from each search are indicated by dotted lines. (Right) Limits on the universal coupling between a leptophobic Z' boson and quarks [17] from various CMS dijet analyses [18]. The expected limits are shown in dashed lines, and the corresponding observed limits are shown in solid lines. The hashed areas show the direction of the excluded area from the observed limits. 
the future, more dark matter scenarios will be studied. An example is the search for trackless jets, which targets SIMPs, strongly interacting DM particles.

\section{Acknowledgments}

The author would like to thank the ATLAS and CMS colleagues for help in preparing this talk, and the ALPS2018 committee for organising this conference so perfectly, in such a privileged location.

\section{References}

[1] G. Aad et al. [ATLAS Collaboration], JINST 3 (2008) S08003. doi:10.1088/1748-0221/3/08/S08003

[2] S. Chatrchyan et al. [CMS Collaboration], JINST 3 (2008) S08004. doi:10.1088/1748-0221/3/08/S08004

[3] A. M. Sirunyan et al. [CMS Collaboration], JHEP 1806 (2018) 120 doi:10.1007/JHEP06(2018)120 [arXiv:1803.06292 [hep-ex]].

[4] G. Altarelli, B. Mele and M. Ruiz-Altaba, Z. Phys. C 45 (1989) 109 Erratum: [Z. Phys. C 47 (1990) 676]. doi:10.1007/BF01552335, 10.1007/BF01556677

[5] J. L. Hewett and T. G. Rizzo, Phys. Rept. 183 (1989) 193. doi:10.1016/0370-1573(89)90071-9

[6] M. Aaboud et al. [ATLAS Collaboration], JHEP 1710 (2017) 182 doi:10.1007/JHEP10(2017)182 [arXiv:1707.02424 [hep-ex]].

[7] M. Aaboud et al. [ATLAS Collaboration], arXiv:1804.03496 [hep-ex].

[8] M. Aaboud et al. [ATLAS Collaboration], arXiv:1801.08769 [hep-ex].

[9] A. M. Sirunyan et al. [CMS Collaboration], JHEP 1801 (2018) 097 doi:10.1007/JHEP01(2018)097 [arXiv:1710.00159 [hep-ex]].

[10] B. A. Dobrescu and F. Yu, Phys. Rev. D 88 (2013) no.3, 035021 Erratum: [Phys. Rev. D 90 (2014) no.7, 079901] doi:10.1103/PhysRevD.88.035021, 10.1103/PhysRevD.90.079901 [arXiv:1306.2629 [hep-ph]].

[11] V. Khachatryan et al. [CMS Collaboration], Phys. Rev. Lett. 117 (2016) no.3, 031802 doi:10.1103/PhysRevLett.117.031802 [arXiv:1604.08907 [hep-ex]].

[12] A. M. Sirunyan et al. [CMS Collaboration], arXiv:1803.08030 [hep-ex].

[13] https://twiki.cern.ch/twiki/bin/view/AtlasPublic/ExoticsPublicResults, ATLAS_DarkMatter_Summary_Vector.pdf

[14] A. Albert et al., arXiv:1703.05703 [hep-ex].

[15] https://twiki.cern.ch/twiki/bin/view/AtlasPublic/ExoticsPublicResults, ATLAS_DarkMatterCoupling_Summary_0labels.pdf

[16] D. Abercrombie et al., arXiv:1507.00966 [hep-ex].

[17] A. M. Sirunyan et al. [CMS Collaboration], Phys. Lett. B 769 (2017) 520 Erratum: [Phys. Lett. B 772 (2017) 882] doi:10.1016/j.physletb.2017.09.029, 10.1016/j.physletb.2017.02.012 [arXiv:1611.03568 [hep-ex]].

[18] https://twiki.cern.ch/twiki/bin/view/CMSPublic/SummaryPlotsEXO13TeV, gq_cms_linearx_logy_LHCP2018.pdf 